\title{
Fabrication of AlN-Based Flexible Piezoelectric Pressure Sensor to Integrate into an Artificial Pancreas ${ }^{\dagger}$
}

\author{
Maria Assunta Signore 1, Chiara De Pascali ${ }^{1}$, Gabriele Rescio ${ }^{1}$, Antonietta Taurino ${ }^{1}$, \\ Paolo Dario ${ }^{2}$, Veronica Iacovacci ${ }^{2}$, Pietro Siciliano ${ }^{1}$, Concetta Martucci ${ }^{1}$, Enrico Melissano ${ }^{1}$, \\ Fabio Quaranta ${ }^{1}$ and Luca Francioso ${ }^{1, *}$ \\ 1 CNR, Institute for Microelectronics and Microsystems, Via Monteroni, 73100 Lecce, Italy; \\ signore@le.imm.cnr.it (M.A.S.); chiara.depascali@le.imm.cnr.it (C.D.P.); gabriele.rescio@le.imm.cnr.it (G.R.); \\ antonietta.taurino@le.imm.cnr.it (A.T.); pietro.siciliano@le.imm.cnr.it (P.S.); \\ mariaconcetta.martucci@cnr.it (C.M.); enrico.melissano@cnr.it (E.M.); fabio.quaranta@cnr.it (F.Q.) \\ 2 The BioRobotics Institute, Scuola Superiore Sant'Anna, Viale R. Piaggio 34, 56025 Pontedera, Italy; \\ paolo.dario@santannapisa.it (P.D.); veronica.iacovacci@santannapisa.it (V.I.) \\ * Correspondence: lucanunzio.francioso@cnr.it; Tel.: +39-0832-422-525 \\ † Presented at the Eurosensors 2018 Conference, Graz, Austria, 9-12 September 2018. \\ Published: 9 November 2018
}

\begin{abstract}
Present work reports the fabrication and characterization of a flexible AlN-based piezoelectric pressure sensor integrated, as insulin capsule punching detector, into an implantable artificial pancreas (AP), developed as automated treatment device for Type 1 diabetes. Ti/AlN/Ti trilayer was sputtered on a thin kapton substrate at room temperature, making the final device flexible and sensitive to the forces range of interest $(0-4 \mathrm{~N})$. The proposed preliminary prototype of $\mathrm{AP}$ comprises a refilling module, interfaced with the intestine wall, able to dock an ingestible insulin capsule. A linearly actuated needle punches the capsule to transfer the insulin to an implanted reservoir. The pressure sensor is located at the connection of the needle with the linear actuator to sense the occurred capsule punching. The sensor waveform output was processed to clearly identify the capsule punching.
\end{abstract}

Keywords: piezoelectric pressure sensor; AlN thin films; implantable artificial pancreas

\section{Introduction}

Modern medical treatments require advanced electronic control functions. In this frame, sensors play a key role as active element of medical devices, both for implanted devices application or for external use, because they can provide data useful to define health states or specific processes performance. In particular, the monitoring of specific automatized processes is very important in the field of implantable artificial organs with the aim to assure the occurred correct operational conditions at the base of the organ working. Implantable flexible pressure sensors represent a class of very versatile devices with a large field of applications in medical field because they can accomplish the tasks to measure pressure in various organs of the body (e.g., brain, eye, heart, bladder) and to evaluate the mechanical pressure in implantable organs where it is necessary. The aim of this work is the realization of a flexible pressure sensor to integrate into an implantable artificial pancreas which comprises a refilling module, interfaced with the intestine wall, able to dock an ingestible insulin capsule. A linearly actuated needle punches the capsule to transfer the insulin to an implanted reservoir. The pressure sensor must be located at the connection of the 
needle with the linear actuator with the aim to sense the occurred punching of the insulin capsule by measuring the punching force.

\section{Materials and Methods}

The Ti/AlN/Ti trilayer has been sputtered from high-purity (99.999\%) $\mathrm{Ti}$ and $\mathrm{Al}$ targets. $\mathrm{Ti}$ layers have been deposited in Ar atmosphere at a pressure of $2.5 \times 10^{-2}$ mbar while AlN in $\left(\mathrm{Ar}+\mathrm{N}_{2}\right)$ mixture at a pressure of $4.3 \times 10^{-3}$ mbar with $\mathrm{N}_{2}$ flux percentage equal to $60 \%$. The plasma was generated by applying an RF power of $150 \mathrm{~W}$ to both targets. All depositions have been performed at room temperature. Ti and AlN films thicknesses were fixed at $150 \mathrm{~nm}$ and $500 \mathrm{~nm}$, respectively.

The structural properties of the films have been investigated by $\mathrm{X}$-ray diffraction measurements (XRD) using $\mathrm{Cu}-\mathrm{K} \alpha$ radiation in the $\theta-2 \theta$ configuration.

Scanning electron microscopy (SEM) was used to investigate the morphology of the specimens. A Zeiss NVISION 40 dual beam FIB machine, equipped with a high resolution SEM Gemini column and an Oxford $350 \mathrm{x}$-act EDS spectrometer, was used for the experiments.

The device has been realized by optimizing conventional cleanroom processes (i.e., photolithography and dry etching techniques) to overcome limitations of flexible substrate integration with IC. To facilitate the fabrication steps, kapton foil has been attached on silicon substrate by a PDMS adhesive layer. After the resist removal, kapton substrate was mechanically detached from silicon support and it has been cut by a metal blade to define the final geometry of the flexible device.

The characterization of the sensor, in terms of generated electric charge under mechanical deformation, was conducted in quasi-static method [1].

\section{Results and Discussion}

\subsection{Structrural and Morphological Characterization of the Piezoelectric Thin Film}

The piezoelectric response of a film of AlN is guaranteed when it grows along c-axis direction, hence structural analysis is aimed to verify the preferential growth of the film along (0002) crystallographic orientation. Figure 1 shows the XRD spectrum of the bilayer AlN/Ti bottom electrode sputtered on kapton substrate.

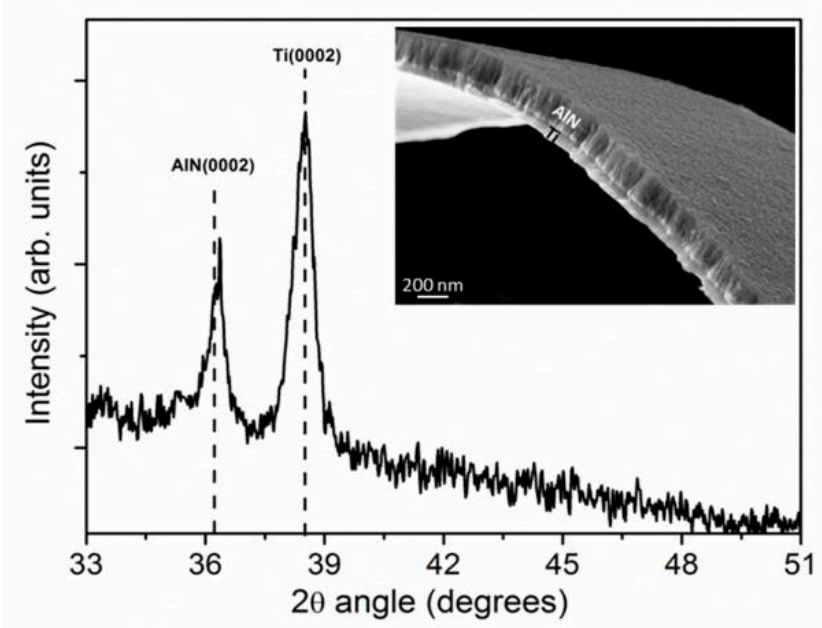

Figure 1. XRD spectrum and SEM image (inset) of Ti/AlN bilayer sputtered on kapton substrate.

It confirms the film growth along c-axis with a slight shift of (0002) diffraction peak towards higher $2 \theta$ angle respect to bulk $2 \theta=36.03^{\circ}$ value, indicating a film growth under tensile stress (in-plain compressive strain) due to unit cell distortion. In the inset, an image obtained by SEM analysis evidences the columnar and dense structure of the sputtered thin films as well as the grain morphology of the surface; moreover, the flexibility of the film deposited on kapton is well 
remarked, without the presence of cracking phenomena, nodal requirement for its integration into the final piezoelectric device.

\subsection{Design and Fabrication of the Piezoelectric Pressure Sensor and Characterization by Quasi-Static Method}

As previously reported, the piezoelectric pressure sensor was designed to be integrated into an implantable artificial pancreas. Figure 2a depicts the preliminary prototype of the artificial organ [2] and the yellow circle defines the area where the pressure sensor must be located.

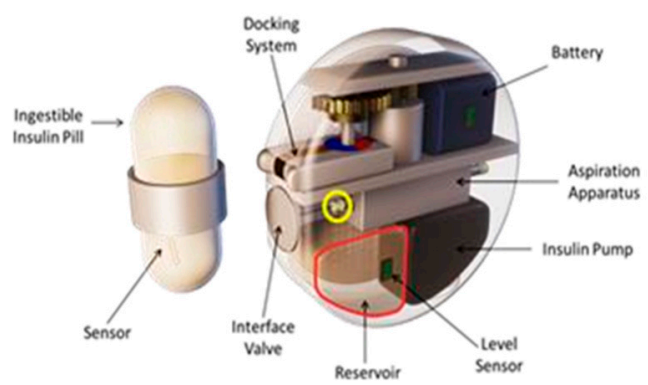

(a)

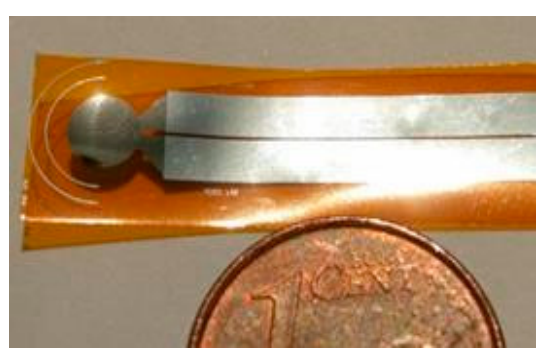

(b)

Figure 2. (a) Preliminary prototype of the implantable artificial pancreas; (b) image of the fabricated piezoelectric pressure sensor on kapton substrate.

As described in Figure 2a, the prototype comprises a refilling module, interfaced with intestine wall, able to dock an ingestible insulin capsule. A linearly actuated needle punches the capsule to transfer insulin to an implanted reservoir. The designed piezoelectric pressure sensor is located at the connection of the needle with the linear actuator to get confirmation of capsule punching by measuring the dynamic force change. Therefore, the circular geometry and the inner and outer diameters of the electrodes have been designed according to the size of the needle-actuator shaft connection (Figure 2b). Preliminary measurements have been performed to verify the piezoelectric response of the designed pressure sensor at the frequency and force range of interest. Figure 3 depicts the electrical output as function of the applied force on the active area of the device at two different frequencies, $1.8 \mathrm{~Hz}$ (a) and $2 \mathrm{~Hz}(\mathrm{~b})$.

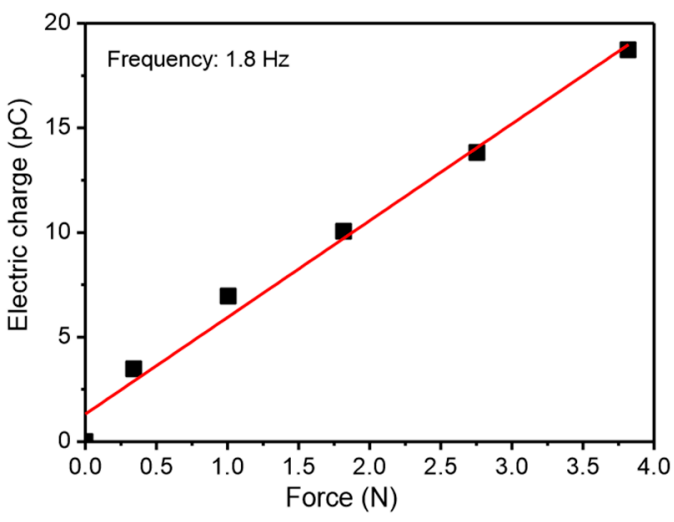

(a)

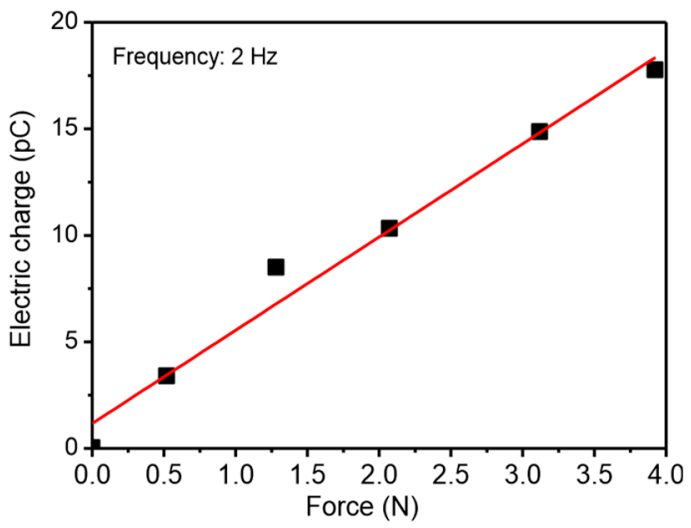

(b)

Figure 3. Electrical output of the piezoelectric pressure sensor as function of the applied force on the active area at different frequencies, (a) $1.8 \mathrm{~Hz}$ and (b) $2 \mathrm{~Hz}$.

The piezoelectric pressure sensor exhibits a promising response when mechanically deformed under loads and frequencies typically involved in the investigated application. The slope of the fit curves provides the evaluation of the $\mathrm{d}_{33}$ piezoelectric constant of AlN thin film by comparison with a reference sample with known piezoelectric coefficient, in the same experimental conditions. This procedure of the quasi-static method permits to obtain a more accurate result. The evaluated $\mathrm{d} 33$ piezoelectric constant value is about $(4.6 \pm 0.3) \mathrm{pC} / \mathrm{N}$, in accordance with the range values reported 
in literature for AlN thin films deposited on rigid substrate $(4.5-5.3 \mathrm{pC} / \mathrm{N})[3,4]$. It should be noted that the piezoelectric coefficient values obtained from thin films deposited on flexible substrates are generally lower than those measured from AlN films realized on rigid substrates (i.e., $2.6 \mathrm{pC} / \mathrm{N} \mathrm{[5]).}$

\section{Conclusions}

A flexible piezoelectric pressure sensor, based on sputtered AlN thin film, has been designed, fabricated and characterized, with the aim to integrate it into an implantable artificial pancreas. The functional characterization of the device, in quasi-static mode, proved the reliability of such fabricated sensor, indicating that it is able to measure dynamic forces up to $4 \mathrm{~N}$ at a frequency range between $1 \mathrm{~Hz}$ and $2 \mathrm{~Hz}$, as requested for the specific application, in a very promising way. This study provides a concept of device design with an easy fabrication method for potential applications in wearable and implantable electronic field.

Acknowledgments: Authors thank the Italian MIUR Ministry for funding the PRIN project "Forget Diabetes: Adaptive Physiological Artificial Pancreas (FORGETDIAB)", which supported present research activity.

Conflicts of Interest: The authors declare no conflict of interest.

\section{References}

1. Fialka, J.; Beneš, P. Comparison of Methods of Piezoelectric Coefficient Measurement. In Proceedings of the Instrumentation and Measurement Technology Conference (I2MTC), Graz, Austria, 13-16 May 2012; pp. 37-42.

2. Iacovacci, V.; Ricotti, L.; Menciassi, A.; Dario, P. The bioartificial pancreas (BAP): Biological, chemical and engineering challenges. Biochem. Pharmacol. 2016, 100, 12-27, doi:10.1016/j.bcp.2015.08.107.

3. Lu, Y.; Reusch, M.; Kurz, N.; Ding, A.; Christoph, T.; Kirste, L.; Lebedev, V.; Zukauskaitè, A. Surface Morphology and Microstructure of Pulsed DC Magnetron Sputtered Piezoelectric AIN and AlScN Thin Films. Phys. Status Solidi A 2018, 215, 1700559, doi:10.1002/pssa.201700559.

4. Fu, Y.Q.; Luo, J.K.; Nguyen, N.T.; Walton, A.J.; Flewitt, A.J.; Zu, X.T.; Li, Y.; McHale, G.; Matthews, A.; Iborra, E.; et al. Advances in Piezoelectric Thin Films for Acoustic Biosensors, Acoustofluidics and Lab on-Chip Applications. Prog. Mater. Sci. 2017, 89, 31-39, doi:10.1016/j.pmatsci.2017.04.006.

5. Akiyama, M.; Morofuji, Y.; Nishikubo, K.; Kamohara, T. Sensitivity enhancement in diaphragms made by aluminum nitride thin films prepared on polyimide films. Appl. Phys. Lett. 2008, 92, 043509, doi:10.1063/1.2839319.

(C) 2018 by the authors; Licensee MDPI, Basel, Switzerland. This article is an open access article distributed under the terms and conditions of the Creative Commons Attribution (CC BY) license (http://creativecommons.org/licenses/by/4.0/). 